\title{
Impacto das infecções relacionadas à assistência à saúde em pacientes acometidos por trauma
}

\section{Impact of health care-related infections in trauma patients}

\author{
Érika Mitiyo Watanabe ${ }^{1}$; Vanessa Fraga de Almeida²; Anali Fernanda Ottunes ${ }^{3}$; \\ Elma Mathias Dessunti ${ }^{4}$; Flávia Meneguetti Pieri ${ }^{5}$; Eleine Aparecida Penha \\ Martins $^{6}$; Gilselena Kerbauy ${ }^{7}$
}

\begin{abstract}
Resumo
As infecções relacionadas à assistência hospitalar impactam negativamente a saúde de pacientes hospitalizados, e refletem em elevados índices de morbimortalidade. Este estudo epidemiológico descritivo teve como objetivo caracterizar as infecções e avaliar seu impacto na saúde de pacientes acometidos por trauma, em um hospital universitário, no período de um ano. Os resultados mostraram que a prevalência das infecções nos pacientes do estudo foi elevada $(15,6 \%)$, acometendo principalmente o sexo masculino (80,0\%), com idade entre 18 e 40 anos (47,5\%), período de internação maior que 15 dias $(78,6 \%)$, mais frequente no trauma fechado $(54,0 \%)$ e nas queimaduras $(32,5 \%)$. Os principais sítios de infecção foram a pneumonia $(49,5 \%)$ seguida pela infecção do trato urinário $(23,8 \%)$. A intubação orotraqueal e o cateterismo vesical de longa permanência estiveram significativamente relacionados à maioria das pneumonias $(60,3 \% ; \mathrm{p}<0,001)$ e infecções do trato urinário $(77,3 \% ; \mathrm{p}<0,001)$, aumentando os riscos para tais infecções em 20 e 6 vezes, respectivamente. A sepse acometeu $44,7 \%$ dos pacientes. Ampla gama de microrganismos apresentou resistência aos antimicrobianos, sendo Acinetobacter baumannii $(92,7 \%, \mathrm{p}<0,001)$ e Klebsiella pneumoniae $(70,5 \%, \mathrm{p}<0,001)$ os mais prevalentes. Evoluíram a óbito $28,8 \%$ dos pacientes, e $96,8 \%$ das mortes relacionavam-se às infecções. A relação das infecções com o óbito foi estatisticamente significativa em pacientes com pneumonia $(37,8 \%, p<0,001)$ e sepse $(54,2 \%, p<0,001)$. A relação das infecções com a maioria dos óbitos evidenciou o impacto negativo desta complicação na saúde das vítimas de traumas.
\end{abstract}

Palavras-chave: Infecção hospitalar. Sepse. Epidemiologia. Causas externas.

\begin{abstract}
Healthcare associated infections have an impact on the health of hospitalized patients and are reflected in high rates of morbidity and mortality. The aim of this descriptive study is to characterize the infections and evaluate their impact on trauma patient health at a University Hospital over a 1-year period. The results showed that the prevalence of infections in trauma patients was elevated (15.6\%), affecting mainly males $(80.0 \%)$, ages between 18 and 30 years $(47.5 \%)$, more than 15 days hospitalization $(78.6 \%)$, more frequent in blunt trauma (54.0\%) and in burns (32.5\%). The principal sites of infection were pneumonia $(49.5 \%)$
\end{abstract}

\footnotetext{
${ }^{1}$ Enfermeira. Residente do Programa de Enfermagem em Cuidados Intensivos da Universidade Estadual de Londrina. Paraná, Brasil.

${ }^{2}$ Aluna da Graduação em Enfermagem da Universidade Estadual de Londrina. Paraná, Brasil.

${ }^{3}$ Aluna da Graduação em Enfermagem da Universidade Estadual de Londrina. Paraná, Brasil.

${ }^{4}$ Doutora em Saúde Pública. Enfermeira. Docente do Departamento de Enfermagem da Universidade Estadual de Londrina, Paraná, Brasil.

${ }^{5}$ Doutora em Enfermagem. Enfermeira. Docente do Departamento de Enfermagem da Universidade Estadual de Londrina, Paraná, Brasil.

${ }^{6}$ Doutora em Enfermagem. Enfermeira. Docente do Departamento de Enfermagem da Universidade Estadual de Londrina, Paraná, Brasil.

${ }^{7}$ Doutora em Microbiologia. Enfermeira. Docente do Departamento de Enfermagem da Universidade Estadual de Londrina, Paraná, Brasil.
} 
followed by urinary tract infection $(23.8 \%)$. The tracheal intubation and long-term vesical catheterization were significantly related to most pneumonias $(60.3 \% ; \mathrm{p}<0.001)$ and urinary tract infection $(77.3 \% ; \mathrm{p}<0.001)$, increasing the risk for such infections on 20 and 6 times, respectively. Sepsis occurred in $44.7 \%$ of patients. A wide range of microorganisms showed resistance to antimicrobials, and Acinetobacter baumannii $192.7 \%$, $\mathrm{p}<0.001)$ and Klebsiella pneumoniae $(70.5 \%, \mathrm{p}<0.001)$ were the most prevalent. $28.8 \%$ of the patients evolved to death, and $96.8 \%$ of deaths were related to infections. The relation between infections and death was statistically significant in pneumonia patients $(37.8 \%, \mathrm{p}<0.001)$ and sepsis $(54.2 \%, \mathrm{p}<0.001)$. The association of infections with the death showed the negative impact of this health complication in trauma patients.

Keywords: Hospital-acquired infection. Sepsis. Epidemiology. External causes.

\section{Introdução}

As infecções relacionadas à assistência à saúde (IRAS) são definidas pelo Ministério da Saúde como qualquer infecção adquirida após a admissão do paciente no serviço de saúde, podendo-se manifestar durante a internação ou após a alta, quando puder ser relacionada com a internação ou com os procedimentos hospitalares (BRASIL, 1998). Refletem em uma problemática que atinge a saúde em níveis mundiais, pois está estritamente relacionada às altas taxas de morbimortalidade, aumento no período de hospitalização e nos custos para o sistema de saúde (OLIVEIRA; DAMASCENO; RIBEIRO, 2009).

Não menos importante, o trauma também repercute em índices de morbimortalidade alarmantes em todo o mundo. Anualmente morrem aproximadamente 5,8 milhões de pessoas em consequência das lesões ocasionadas pelo trauma, e muitas outras ficam debilitadas, principalmente nos países de baixa renda e de renda média, onde mais de $90 \%$ das mortes são decorrentes do trauma (WORLD HEALTH ORGANIZATION, 2009). No Brasil, as causas externas foram responsáveis por 145.842 óbitos no ano de 2011 (BRASIL, 2014).

As lesões do trauma, associadas à quebra do mecanismo de defesa imunológica e colonização por microrganismos patogênicos do ambiente hospitalar, favorecem os riscos para as infecções. A quebra na barreirainata, decorrentedainterrupção daintegridade da pele, a formação de hematomas e/ou presença de corpos estranhos ocasionados pelos mecanismos de trauma ou por meio dos procedimentos invasivos e incisões cirúrgicas permitem a invasão pelos microrganismos e favorecem o desenvolvimento das IRAS em pacientes acometidos por trauma (ZILIOTTO, 2007).

Adicionalmente, outro fator complicador relacionado às IRAS é a ampla resistência bacteriana aos antimicrobianos. As IRAS causadas por esses patógenos resistentes às opções terapêuticas estão relacionadas a custos elevados na assistência, períodos prolongados de hospitalização e aumento na mortalidade, tendo em vista as limitações terapêuticas inerentes a estes microrganismos (CENTERS FOR DISEASE CONTROL AND PREVENTION, 2013).

No intuito de compreender o impacto das IRAS na saúde de pacientes vítimas de trauma, este estudo teve como objetivo caracterizar as IRAS, avaliar o seu impacto na saúde e identificar os riscos destas infecções para o óbito de pacientes acometidos por trauma, atendidos em um hospital escola.

\section{Método}

Estudo realizado no Hospital Universitário de Londrina (HU-UEL), órgão suplementar da Universidade Estadual de Londrina, entidade que se dedica ao ensino, pesquisa e extensão de serviços à comunidade, por meio da prestação de atendimento universal. É um hospital terciário, centro de referência para o Sistema Único de Saúde (SUS) na região norte do estado do Paraná, Brasil. Atende pacientes de 
cerca de 250 municípios do Paraná e de mais de 100 cidades de outros estados. Possui o total de 353 leitos distribuídos em unidades de internamento, prontosocorro, unidades de terapia intensiva ao adulto (UTI I e UTI II), criança e neonatos. Somado aos setores hospitalares, o HU-UEL é referência no tratamento de queimados para o norte do estado. $\mathrm{O}$ centro de tratamento de queimados (CTQ) é composto por enfermaria, UTI e centro cirúrgico.

Trata-se de um estudo epidemiológico, retrospectivo, de abordagem descritiva, cuja população foi composta por todos os prontuários dos pacientes com idade igual ou superior a 18 anos, diagnosticados com algum tipo de trauma, que adquiriram IRAS durante internação no HU-UEL no ano de 2010.

Foram adotados os seguintes critérios de inclusão: pacientes com diagnósticos de lesão, e alguma outra consequência de causas externas de acordo com a Classificação Internacional de Doenças, CID-10, capítulo XIX, que evoluíram com IRAS de acordo com critérios do Centers for Disease Control and Prevention/National Healthcare Safety Network - CDC/NHSN (HORAN; ANDRUS; DUDECK, 2008). Quanto ao desenvolvimento de sepse, esta complicação foi classificada como: sepse, sepse grave e choque séptico de acordo com critérios do International Sepsis Definitions Conference - 2001 (LEVY et al., 2003).

Os dados relacionados ao sexo, idade, tempo de internação, comorbidades, tipo de trauma e área corpórea acometida, principal sitio de infecção, desenvolvimento de sepse, procedimentos invasivos e desfecho (alta ou óbito) foram coletados pelos autores do trabalho a partir dos prontuários dos pacientes e das fichas de notificação de IRAS, formuladas de acordo com critérios do National Nosocomial Infection Surveillance, USA - NNIS (EMORI et al., 1991) e preenchidas pela equipe multiprofissional da Comissão de Controle de Infecção Hospitalar (CCIH) da instituição.

Os resultados das culturas microbiológicas foram extraídos do banco de dados disponibilizados pelo serviço de informática da instituição. Foram classificados como multirresistentes aos antimicrobianos Acinetobacter baumannii e Pseudomonas sp resistentes aos carbapenêmicos; Klebsiella pneumoniae e Escherichia coli resistentes a carbapenem ou cefalosporinas (terceira ou quarta geração) ou monobactâmicos; Staphylococcus coagulase negativa e Staphylococcus aureus resistentes a oxacilina; Enterobacter sp, outras Enterobactérias e Serratia $s p$ resistentes a carbapenêmicos ou cefalosporina (quarta geração); Enterococcus $s p$ resistente a vancomicina e Streptococcus sp resistente a penicilina, cefotaxima, levofloxacina, carbapenêmicos, glicopeptídeos e macrolídeos; Burkholderia cepacia, Stenotrophomonas sp e Moraxella $s p$ foram agrupadas como não-fermentadores e todas foram consideradas multirresistentes devido à resistência intrínseca a vários antibióticos (SIEVERT et al., 2013).

As análises estatísticas foram realizadas no software Statistical Package for the Social Sciences (SPSS) versão 19. A descrição das variáveis foi realizada por meio de médias, desvio-padrão, proporções e distribuição das frequências das variáveis. Para avaliar as associações das variáveis com o óbito foi realizada análise bivariada por meio do teste Quiquadrado $\left(\mathrm{X}^{2}\right)$ ou o teste exato de Fisher. Como medidas de força de associações, foram utilizados o risco relativo e intervalos de confiança de 95\% (IC 95\%). O nível de significância adotado foi de $5 \%(\alpha=0,05)$.

Este estudo foi aprovado pelo Comitê de Ética em Pesquisa envolvendo Seres Humanos da Universidade Estadual de Londrina e responde com Certificado de Apresentação para Apreciação Ética (CAAE) número 0015.0.268.000-11.

\section{Resultados e Discussão}

No período estudado foram admitidos 1380 pacientes acometidos por trauma no HU-UEL. Desse total, $215(15,6 \%)$ adquiriram pelo menos uma infecção durante o período de hospitalização, representando a população do estudo. Estudos 
evidenciam que a prevalência das IRAS em pacientes traumatizados oscila entre 9,2\% e 42\% (CARICATO et al., 2009; PORIES et al., 2001).

A tabela 1 apresenta a caracterização da população, destacando o predomínio do sexo masculino $(80,0 \%)$ e faixa etária entre 18 e 40 anos (47,5\%), com uma média de idade de 47 anos. Resultados semelhantes foram constatados em estudo brasileiro realizado na cidade de Curitiba-PR, no qual 84,6\% dos 416 pacientes com diagnóstico de trauma e IRAS também eram do sexo masculino, com média de idade de 32 anos (GIAMBERARDINO et al., 2007). Em outra pesquisa, realizada em um hospital universitário da Tailândia, a idade média também foi de adultos jovens, 29 anos (JAMULITRAT; NARONG; THONGPIYAPOOM, 2002). Podemos relacionar esses dados provavelmente ao fato de que os homens na faixa etária economicamente ativa estão mais expostos aos acidentes decorrentes de causas externas, principalmente por acidentes de transporte terrestre que respondem por $26,5 \%$ dos óbitos no Brasil (BRASIL, 2010). As causas externas são a principal causa de morte na população de 40 a 59 anos e o risco entre os homens é quase cinco vezes maior em relação às mulheres (32,6 óbitos e 7,1 óbitos por 100 mil homens) respectivamente (BRASIL, 2010).

Em relação ao período de internação, a média de permanência hospitalar foi de 30,3 dias (intervalo de 2-135 dias), sendo que $78,6 \%$ dos pacientes permaneceram internados por mais de 15 dias. Estudo tailandês também apresentou internação de longa permanência, aproximadamente 20,3 dias (JAMULITRAT; NARONG; THONGPIYAPOOM, 2002). Em contrapartida, o período médio de hospitalização para pacientes que não desenvolvem IRAS é de 3,7 dias (OLIVEIRA; KOVNER; SILVA, 2010), evidenciando o impacto negativo das IRAS no prolongamento da hospitalização.

Dentre os pacientes, 5,1\% possuíam comorbidades associadas ao diagnóstico. Provavelmente esse percentual é baixo devido ao perfil da população acometida pelo trauma ser mais jovem.
A diabetes mellitus foi a comorbidade mais prevalente $(36,3 \%)$, acometendo pacientes com idade entre 67 a 87 anos. Estudo norte-americano realizado com 10.555 pacientes admitidos no Shock Trauma Center em Maryland, Estados Unidos, identificou que os pacientes com idade inferior a 65 anos e que apresentavam diabetes mellitus representavam apenas 3,1\% do total da amostra e, destes, 29\% adquiriram infecção. Já em pacientes com idade superior a 65 anos a diabetes esteve presente em mais da metade (51\%) dos casos de IRAS (BOCHICCHIO et al., 2001).

As lesões mais frequentes em pacientes que desenvolveram IRAS foram o trauma fechado $(54,0 \%)$, seguido pelas queimaduras $(32,6 \%)$ e pelo trauma aberto $(13,5 \%)$. Dentre as queimaduras, o grande queimado foi mais frequentemente acometido pelas IRAS (78,5\%). Esse resultado corrobora com o de outros estudos, nos quais $74,5 \%$ a $86,0 \%$ das vítimas de trauma que desenvolveram IRAS sofreram traumas fechados (GIAMBERARDINO et al., 2007; PAPIA et al., 1999).

Quanto a área corpórea acometida pelo trauma, houve predomínio dos membros inferiores $(22,3 \%)$ e múltiplas lesões (17,2\%). Trauma acometendo o crânio (72,3\%), as extremidades $(36,2 \%)$ e o tórax $(28,7 \%)$ foram mais frequentes em pacientes com IRAS em estudo realizado na Tailândia (JAMULITRAT; NARONG; THONGPIYAPOOM, 2002). Lesões nas extremidades seguidas por lesões cranianas também foram as mais frequentes em Burlington-USA (PORIES et al., 2001).

As lesões nas extremidades, principalmente nos membros inferiores e o trauma em múltiplas localizações, podem restringir a movimentação dos pacientes e aumentar o tempo de permanência no leito. A imobilização reflete diretamente no sistema pulmonar, pois diminui o movimento diafragmático e a excursão torácica, com redução da ventilação, o estímulo da tosse e a eliminação das secreções. O acúmulo de secreções nas bases pulmonares favorece a proliferação bacteriana, que contribui 
com o desenvolvimento da pneumonia (CAZEIRO; PERES, 2010).

Tabela 1 - Frequência dos pacientes acometidos por trauma que desenvolveram IRAS segundo variáveis sociodemográficas e clínicas. Londrina, 2012

\begin{tabular}{lll}
\hline VARIÁVEIS & N & $\%$ \\
\hline SEXO
\end{tabular}

\section{Sexo}

Feminino

Masculino 43 172 20,0

\section{Idade}

18 a 30 anos

31 a 40 anos

64

41 a 50 anos

\section{8}

51 a 60 anos

61 a 70 anos

71 a 80 anos

Acima de 80 anos

\section{1}

27

Período de Hospitalização

0-5 dias

6-14 dias

15-30 dias

$>30$ dias

\section{Comorbidade}

Sim

$$
11
$$

Não

\section{Classificação do Trauma}

Aberto

Fechado

Queimadura

\section{Classificação da Queimadura}

Pequeno Queimado

Médio Queimado

Grande Queimado

Região Corpórea Acometida pelo

Trauma*

Toraco-abdominal

Cabeça-pescoço

Coluna vertebral

Membro Inferior

Membro Superior

Politrauma

*Trauma aberto e fechado.

Fonte: Autores

$14 \quad 6,5$

$55 \quad 25,6$

Um total de 315 infecções foram diagnosticadas nos 215 pacientes estudados. A maioria dos pacientes, $140(65,1 \%)$ apresentaram apenas um episódio de infecção, enquanto 17,2\% (37) foram diagnosticados com dois episódios de infecção e $17,7 \%$ (38) com três ou mais episódios.

Os principais sítios infecciosos observados no atual estudo foram o pulmão (49,5\%), seguido pelo trato urinário $(23,8 \%)$ e sítio cirúrgico $(10,5 \%)$, apresentados na figura 1. Corroborando com esses dados, pesquisa canadense realizada com 563 pacientes, mostrou predomínio da pneumonia, $28,0 \%$, seguida da infecção do trato urinário (ITU), $24,0 \%$ e do sítio cirúrgico, 18,0\% (PAPIA et al., 1999). Estudo italiano evidenciou as infecções da corrente sanguínea $(13,7 \%)$ dentre as mais prevalentes em pacientes acometidos por trauma (CARICATO et al., 2009).

Dados de IRAS na população geral mostram predomínio também das pneumonias, mas evidenciam também as infecções da corrente sanguínea. E trazem as infecções de sítio cirúrgico como as de menor frequência (NOGUEIRA et al., 2009; SIEVERT et al., 2013).

A sepse esteve presente em quase a metade dos pacientes $(44,7 \%)$ e, em relação à classificação desta complicação, o choque séptico representou a maioria $(73,0 \%)$, seguido pela sepse $(24,0 \%)$ e sepse grave $(3,0 \%)$.

Figura 1 - Frequência dos sítios infecciosos em pacientes acometidos por trauma que desenvolveram IRAS. Londrina, 2012

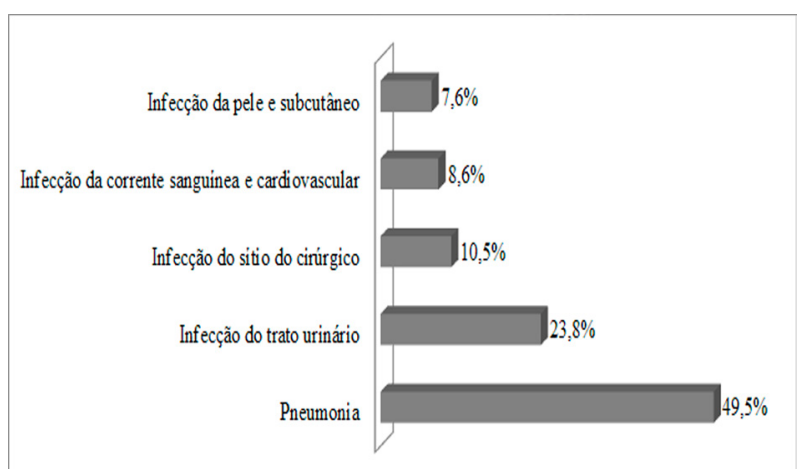

Fonte: Autores. 
Em relação aos procedimentos invasivos, a sua relização pode inativar as barreiras de defesas naturais do hospedeiro, favorecendo a entrada de microrganismos. Um importante exemplo é a intubação orotraqueal, que impedem os mecanismos de defesa inata, como a motilidade ciliar e o reflexo da tosse, favorecendo os fatores de patogenicidade microbianos (LACHERADE et al., 2010).

A intubação orotraqueal foi realizada em 45,6\% (98) dos pacientes e esteve significativamente relacionada à maioria das pneumonias (60,3\%; $\mathrm{p}<0,001$ ), o que aumenta em 20 vezes o risco para essa infecção se comparado aos não intubados. Outra pesquisa aponta que os riscos de adquirir pneumonia aumentam de 6 a 21 vezes em pacientes intubados (CRUZ et al., 2011).

No presente estudo, a cateterização vesical de longa permanência foi realizada na metade dos pacientes $(50,2 \%)$ e apresentou relação estatisticamente significativa com a ITU (77,3\%; $\mathrm{p}<0,001$ ), aumentando em 6 vezes o risco dessa infecção em pacientes cateterizados.

Em um estudo realizado com 5.736 pacientes vítimas de trauma, 47,0\% (2.710 pacientes) utilizaram a cateterização vesical de longa permanência, entretanto, uma porcentagem menor de pacientes, $17,9 \%$, desenvolveram infecção do trato urinário (MONAGHAN et al., 2011).

A cateterização venosa central esteve presente em $17,2 \%$ da população e foi relacionada a $42,9 \%$ das infecções da corrente sanguínea. Essas infecções ocorrem principalmente pelo uso de cateteres vasculares centrais e em associação com outros cateteres são responsáveis por cerca de $60 \%$ das bacteremias hospitalares (ANVISA, 2013).
Os pacientes que se submeteram a procedimentos cirúrgicos foram a minoria $(13,4 \%$; $n=29)$ em relação aos que realizaram apenas o tratamento clínico (86,5\%; n=186). Entretanto, as infecções desse sítio acometeram $13,9 \%$ dos pacientes que passaram por esses procedimentos, representando 9,7\% dos sítios de infecção diagnosticados na população do estudo.

Em relaçãoaoperfilmicrobiológicodasinfecçõesem pacientes acometidos por trauma, 328 microrganismos foram isolados em culturas positivas de sangue, urina e secreções traqueais. Os patógenos mais prevalentes foram os Gram-negativos: Acinetobacter baumannii (16,8\%; 55), Klebsiella pneumoniae $(13,4 \% ; 44)$ e Pseudomonas aeruginosas $(12,8 \% ; 42)$. Em estudo que avaliou 290 IRAS em pacientes traumatizados, os microrganismos isolados que mais prevaleceram foram Staphylococcus coagulase negativa $(21,8 \%)$, A.baumannii (20,4\%) e Staphylococcus aureus (10,5\%) (GIAMBERARDINO et al., 2007). Já a pesquisa realizada em um hospital universitário com pacientes traumatizados durante o período de três anos, demonstrou que os patógenos mais frequentes foram $S$. aureus (19,7\%), P. aeruginosas (19,1\%), $K$. pneumoniae e $A$. baumannii (18,0\%) (JAMULITRAT; NARONG; THONGPIYAPOOM, 2002).

Os microrganismos multirresistentes (MR) representaram quase metade, $48,8 \%$, do total de isolados, com predomínio de A. baumannii (92,7\%, $\mathrm{p}<0,001)$ e $K$. pneumoniae $(70,5 \%, \mathrm{p}<0,001)$, de acordo com a Tabela 2. Resultados semelhantes foram reportados por um estudo brasileiro realizado com pacientes com diferentes disagnósticos, internados em UTI, no qual a multirresistência prevaleceu entre A. baumannii $(35,1 \%)$, P. aeruginosa $(21,6 \%), S$. aureus, K. pneumonia e E. coli (10,8\%) (OLIVEIRA; KOVNER; SILVA, 2010). 
Tabela 2 - Frequência dos microrganismos multirresistentes isolados em pacientes acometidos por trauma que desenvolveram IRAS. Londrina, 2012

\begin{tabular}{lcccc}
\hline \multicolumn{1}{c}{ Microrganismos } & Total & & & Valor p \\
\cline { 3 - 4 } & & $\mathbf{N}$ & $\mathbf{\%}$ & \\
\hline Acinetobacter baumannii & 55 & 51 & 92,7 & $<0,001$ \\
Klebsiella pneumoniae & 44 & 31 & 70,5 & $<0,001$ \\
Não fermentadores & 9 & 9 & 100 & $<0,001$ \\
Staphylococcus coagulase negativa & 24 & 15 & 62,5 & $<0,001$ \\
Staphyloccus aureus & 32 & 19 & 59,4 & $<0,001$ \\
Enterobacter sp & 28 & 15 & 53,6 & $<0,001$ \\
Outras Enterobactérias & 37 & 19 & 51,4 & $<0,001$ \\
Pseudomonas sp & 42 & 17 & 40,5 & $<0,001$ \\
Echerichia coli & 24 & 8 & 33,3 & - \\
Enterococcus sp & 19 & 1 & 5,3 & - \\
Streptococcus sp & 7 & 0 & 0 & - \\
Serratia sp & 7 & 0 & 0 & - \\
TOTAL & $\mathbf{3 2 8}$ & $\mathbf{1 8 2}$ & $\mathbf{5 5 . 5}$ & \\
\hline Fonte: Autors & & &
\end{tabular}

Fonte: Autores.

Os óbitos ocorreram em 28,8\% (62) dos pacientes e as IRAS contribuíram de alguma forma para a maioria dessas mortes $(96,8 \%)$. A relação das IRAS com óbitos de pacientes acometidos por trauma também foi observada em outras instituições, contudo, contribuiram para um menor número de mortes. Em estudo realizado em uma unidade de trauma canadense, dos 31 pacientes que evoluíram a óbito, 15 (48,3\%) adquiriram infecção, sendo que em quatro pacientes a infecção foi a causa direta do óbito (PAPIA et al., 1999). Em pesquisa norte americana, a mortalidade foi significativamente mais elevada em pacientes com IRAS (10\%) se comparada a pacientes que não desenvolveram infecção (2\%) (PORIES et al., 2001).

Quanto aos riscos de óbito para pacientes traumatizados que desenvolveram IRAS, a pneumonia apresentou associação estatística significativa $(37,8 \%, \mathrm{p}<0,001)$, aumentando em 11 vezes o risco de morrer. A sepse foi diagnosticada em $54,2 \%$ dos pacientes que evoluíram a óbito $(p<0,001)$, e aumentou em quase 13 vezes o risco de morrer em relação àqueles que não desenvolveram essa complicação. Em estudo norte-americano, os pacientes vítimas de trauma com sepse apresentaram 6 vezes mais risco de morrer em comparação aos pacientes sem infecção (GLACE et al., 2011).

\section{Conclusão}

O presente estudo identificou o perfil epidemiológico das IRAS em pacientes acometidos por traumas e constatou seu impacto negativo 
na saúde desses pacientes, tendo em vista a contribuição na mortalidade. As IRAS foram relacionadas a 96,8\% dos casos que evoluíram a óbito. A pneumonia e a sepse aumentaram em onze e doze vezes, respectivamente, o risco de óbito em comparação aos pacientes que não adquiriram essas infecções. Os microrganismos que apresentaram resistência aos antibióticos representaram 48,8\% da amostra e os patógenos mais prevalentes foram o $A$. baumannii e K. pneumoniae.

A relevância deste estudo deu-se pela caracterização das IRAS em pacientes acometidos por trauma, elucidação dos fatores de risco para o desenvolvimento destas infecções e relação com o óbito.

Diante deste contexto, destaca-se a necessidade da implementação de estratégias de prevenção das infecções e esforços que visem à melhoria da qualidade assistencial voltada a pacientes vítimas de trauma. Portanto, ações de vigilância epidemiológica, monitoramento dos casos que apresentam riscos para IRAS e controle rigoroso dos procedimentos assistenciais são ferramentas que podem contribuir no controle das IRAS e, consequentemente, os óbitos a elas relacionados.

\section{Referências}

AGÊNCIA NACIONAL DE VIGILÂNCIA SANITÁRIA - ANVISA. Segurança do paciente e qualidade em serviços de saúde. Medidas de prevenção de infecção: relacionada à assistência à saúde. Brasília: Anvisa, 2013.

BOCHICCHIO, G. V.; JOSHI, M.; KNORR, K.; SCALEA, T. M. Impact of nosocomial infections in trauma: does age make a difference? The Journal of Trauma, Baltimore, v. 50, n. 4, p. 612-619, 2001.

BRASIL. Ministério da Saúde. Estatísticas de mortalidade: óbitos por ocorrência segundo causas externas do Brasil, 2011. Disponível em: <www2.datasus.gov.br/DATASUS/ index. php? area $=0205 \& \mathrm{VObj}=\mathrm{http}: / /$ tabnet.datasus.gov. br/cgi/deftohtm.exe?sim/cnv/ext10>. Acesso em: 5 jul. 2014.
Portaria n. 2.616, de 12 de maio de 1998. $\overline{\text { Expede }}$ diretrizes e normas para a prevenção e o controle das infecções hospitalares. Diário Oficial da República Federativa do Brasil, Brasília, seção1, 13 mai.1998.

Secretaria de Vigilância em Saúde. Epidemiologia das causas externas no Brasil: mortalidade por acidentes e violências no período de 2000 a 2009. Brasília: Ministério da Saúde, 2010 .

CARICATO, A.; MONTINI, L.; BELLO, G.; MICHETTI, V.; MAVIGLIA, R.; BOCCI, M. G.; MERCURIO, G.; MEGGIORE, S. M.; ANTONELLI, M. Risk factors and outcome of Acinetobacter baumanii infection in severe trauma patients. Intensive Care Medicine, New York, v. 35, n. 11, p. 1964-1969, 2009.

CAZEIRO, A. P. M.; PERES, P. T. A terapia ocupacional na prevenção e no tratamento de complicações decorrentes da imobilização no leito. Cadernos de Terapia Ocupacional da UFSCar, São Carlos, v. 18, n. 2, p. 149-167, 2010.

CENTERS FOR DISEASE CONTROL AND PREVENTION. Antibiotic resistance threats in the united states. Atlanta: CDC, 2013.

CRUZ, F. L. C.; MENESES, M. R. R.; SERRA, S. R.; BARBOSA, M. C. G. Pneumonia associada a ventilação mecânica: medidas preventivas. Revista de Pesquisa em Saúde, São Luís, v. 12, n. 1, p. 5659, 2011.

EMORI, T. G.; CULVER, D. H.; HORAN, T. C.; JARVIS, W. R.; WHITE, J. W.; OLSON, D. R.; BANERJEE, S.; EDWARDS, J. R.; MARTONE, W.; GAYNES, R. P. National nosocomial infections surveillance system (NNIS): description of surveillance methods. American Journal of Infection Control, Nova York, v. 19, n. 1, p. 19-35, 1991.

GIAMBERARDINO, H. I. G.; CESÁRIO, E. P.; CARMES, E. R.; MULINARI, R. A. Risk factors for nosocomial infection in trauma patients. Brazilian Journal of Infectious Diseases, Salvador, v.11, n. 2, p. 285-289, 2007. 
GLANCE, L. G.; STONE, P. W.; MUKAMEL, D. B.; DICK, A. W. Increases in mortality, length of stay, and cost associated with hospital-acquired infections in trauma patients. Archives of Surgery, Chicago, v.146, n. 7, p. 794-801, 2011.

HORAN, T. C.; ANDRUS, M.; DUDECK, M. CDC/NHSN Surveillance definition of health careassociated infection and criteria for specific types of infections in the acute care setting. American Journal of Infection Control, Saint Louis, v. 36, n. 5, p. 309-332, 2008.

JAMULITRAT, S.; NARONG, M. N.; THONGPIYAPOOM, S. Trauma severity scoring systems as predictors of nosocomial infection. Infection Control and Hospital Epidemiology, Chicago, v. 23, n. 5, p. 268-273, 2002.

LACHERADE, J. C.; JONGHE, B.; GUEZENNEC, P.; DEBBAT, K.; HAYON, J.; MONSEL, A.; FANGIO, P.; VECCHI, C. A.; RAMAUT. C.; OUTIN, H.; BASTUJI-GARIN, S. Intermittent subglottic secretion drainage and ventilator-associated pneumonia. American journal of respiratory and critical care medicine, Nova York, v.182, n. 7, p. 910-917, 2010.

LEVY, M. M.; FINK, M. P.; MARSHALL, J. C.; ABRAHAM, E.; ANGUS, D.; COOK, D.; COHEN, J.; OPAL, S. M.; VINCENT, J. L.; RAMSAY, G. 2001 SCCM/ESICM/ACCP/ATS / SIS International Sepsis Definitions Conference. Critical Care Medicine, Nova York, v. 31, n. 4, p. 1250-1256, 2003.

MONAGHAN, S. F.; HEFFERNAN, D. S.; THAKKAR, R. K.; REINERT, S. E.; MACHAN, J. T.; CONNOlly, M. D.; GREGG, S. C.; KOZLOFF, M. S.; ADAMS, C. A.; CIOFFI, W. $\mathrm{G}$. The development of a urinary tract infection is associated with increased mortality in trauma patients. The Journal of Trauma, Baltimore, v. 71, n. 6, p. 1569-1574, 2011.

NOGUEIRA, P. S. F.; MOURA, E. R. F.; COSTA, M. M. F.; MONTEIRO, W. M. S.; BRONDI, L. Perfil da infecção hospitalar em um hospital universitário. Revista de Enfermagem UERJ, Rio de Janeiro, v.17, n. 1, p. 96-101, 2009.
OLIVEIRA, A. C.; DAMASCENO, Q. S.; RIBEIRO, S. M. P. Infecções relacionadas à assistência em saúde: desafios para a prevenção e controle. REME: Revista Mineira de Enfermagem, Belo Horizonte, v. 13, n. 3, p. 445-450, 2009.

OLIVEIRA, A. C.; KOVNER, C. T.; SILVA, R. S. Nosocomial Infection in an Intensive Care Unit in a Brazilian University Hospital. Revista Latino-Americana de Enfermagem, São Paulo, v. 18, n. 2, p. 233-239, 2010.

PAPIA, G., MCLELLAN, B. A.; EL-HELOU, P.; LOUIE, M.; RECHLIS, A.; SZALAI, J.; SIMOR, A. E. Infection in hospitalized trauma patients: incidence, risk factors and complications. The Journal of Trauma, Baltimore, v. 47, n. 5, p. 923-937, 1999.

PORIES, S. E.; GAMELLI, R. L.; MEAD, P. B.; GOODWIN, G.; HARRIS, F. V. P. The epidemiologic features of nosocomial infections in patients with trauma. Archives of Surgery, Chicago, v. 126, n. 1, p. 97-99, 2001.

SIEVERT, D. M.; RICKS, P.; EDWARDS, J. R.; SCHNEIDER, A.; PATEL, J.; SRINIVASAN, A.; KALlEN, A. M. D.; LIMBAGO, B. P. H. D.; FRIDKIN, S. M. D. Antimicrobial-resistant pathogens associated with heathcare-associated infections: summary of data reported to the National Heathcare safety Network at the Centers for Disease Control and Prevention, 2009-2010. Infection Control and Hospital Epidemiology, Chicago, v. 34, n. 1, p. 1-14, 2013.

WORLD HEALTH ORGANIZATION. Guidelines for trauma quality improvement programmes. Geneva: WHO, 2009. Disponível em: $\quad<$ http://whqlibdoc.who.int/publications/ 2009/9789241597746_eng.pdf>. Acesso em: 03 jul. 2014.

ZILIOTTO, J. A. Infecção em cirurgia de emergências e trauma: prevenção, diagnóstico e tratamento. Medicina, Ribeirão Preto, v. 40, n. 3, p. 329-334, 2007.

Recebido em: 14 jul. 2014. Aceito em: 05 jan. 2015. 
